\title{
Erratum to: Grammar Induction by Unification of Type-logical Lexicons
}

\author{
Sean A. Fulop
}

Published online: 25 November 2010

(C) Springer Science+Business Media B.V. 2010

\section{Erratum to: J Log Lang Inf (2010) 19:353-381 DOI 10.1007/s10849-009-9108-7}

This corrigendum corrects an inaccuracy in the paper that appeared in JoLLI volume 19, pp. 353-381. The problem originates in the definition and proposition that are now quoted:

Definition 4 A type logic is said to be structurally deterministic just in the case that for any provable sequent $\Gamma \Rightarrow C$ in which $\Gamma$ contains no structural modal environment, there is no other provable sequent $\Gamma^{\prime} \Rightarrow C$ in which $\Gamma^{\prime}$ is a reconfiguration of $\Gamma$ (i.e. a different tree structure over precisely the same multiset of formula occurrences).

Proposition 5 Every permitted logic is structurally deterministic.

This proposition is not true, because the above definition of structurally deterministic is too broad. The correction is to restrict the form of the "provable sequent" $\Gamma \Rightarrow C$ in the definition using the following conditions: (i) formula $C$ contains no slash operators; (ii) G-term $\Gamma$ has no types repeated, and has no subtypes repeated within any one type. With these restrictions, the proposition is correct, and its proof in the original paper says nothing incorrect. Proposition 5 was used in the paper to prove Proposition 11 therein, and the above narrowing of Proposition 5 actually makes it more obviously

The online version of the original article can be found under doi:10.1007/s10849-009-9108-7.

S. A. Fulop $(\varangle)$

Department of Linguistics, California State University Fresno, 5245 N. Backer Ave. PB92,

Fresno, CA 93740-8001, USA

e-mail: sfulop@csufresno.edu 
applicable for this purpose. It is therefore not necessary to modify Proposition 11 or its proof. The author would like to thank the two anonymous referees for Mathematical Logic Quarterly for pointing out this flaw. 